\title{
A MODULAR APPROACH TO STORY GENERATION
}

\author{
Lyn Pemberton \\ School of Cognitive and Computing Sciences \\ University of Sussex \\ Falmer \\ Brighton \\ BN1 9QN \\ United Kingdom
}

\begin{abstract}
One way of characterising texts is in terms of the discourse structures on which they appear to be built. Each type of text, or genre, e.g. the sports report, the recipe, the sermon, the proverb, will have associated with it a characteristic organisation of units. In this paper, a general model of the structure of one text type, the story, is described. This model forms the basis of a program which combines the general story structure principles with rules governing a particular sub-genre, the Old French epic, in order to generate story summaries.
\end{abstract}

\section{INTRODUCTION}

Over the last twelve years, research in Psychology and AI into narrative structure has been marked by acrimonious disputes over the right to existence of the "story grammar" approach. The point at issue is whether it is possible, as story grammarians suggest, to identify structural regularities particular to narrative texts (Mandler and Johnson, 1980; Pemberton, 1987; Shen, 1988), or whether any structural regularities should be interpreted rather as world knowledge, usable in contexts other than stories (Garnham, 1983; Wilensky, 1980; Yazdani, 1982).

The work described in this paper adopts the story grammarian position, arguing that a general model of story structure may be used, in conjunction with, but separate from, a model of the "story world", to generate stories. Moreover, a fuller version of such an account of story generation must draw not only on the general story structure model and the story world model, but also on aspects of the audience, the author, the medium of expression and the cultural context.

\section{TEXT TYPES}

People are able to categorise texts into types, according to the global conditions of coherence which they perceive at work in texts. Knowledge of the particular structures of each text type is an element of the cultural competence of the speaker/hearer, enabling $\mathrm{him} / \mathrm{her}$ to process varieties of cultural artefact such as jokes, sermons, weather reports, sets of instructions and so on, in appropriate ways (Ryan, 1981).

Often, especially in "realistic" texts, it may seem that these conditions of coherence are reducible to the everyday concepts which we use to analyse aspects of the real world, especially notions such as plans, goals and intentions. On the other hand, the fact that the same real world events, e.g. a road accident, or a bank robbery, may be represented in texts of widely different types, such as a newspaper article, a telegram message, a joke, or a conversational anecdote, indicates that structuring models, in addition to those imposed by the structure of the content, are at work in texts. Such models facilitate the 
processing of texts, creating certain expectations when we recognise a text as an instance of a particular genre, and providing a set of pattems to guide the creation of new instances.

Text types interact in complex ways with the other categorial features of texts, such as content, speaker type, speech situation, register and speech act identity. Sometimes, text types are highly constrained in this regard. For example, conventionally a text of the "sermon" genre is uttered in the "church service" speech situation, in a formal register: it is normally produced only by a priest-figure, and often serves as a "warning" or "exhortation" speech act. Texts of the story genre are not tightly linked to any such characteristics: they may be expressed by anyone, in any register, in almost any speech situation, and, while their illocutionary force is essentially "assertion", their intended perlocutionary forces may be many and varied. However, sub-genres such as the detective story, the narrative ballad, the traditional folk tale and so on, will impose further constraints of their own.

\section{A MODEL OF STORY GENERATION}

As this discussion of genre theory implies, story structure cannot be discussed in isolation from a variety of other constraints. A theory of story structure for the purposes of text generation must be situated within a broader theory of story production, which can perhaps best be described in terms of the sorts of knowledge which contribute to the creation of a story. A full story generating system would need access to many different kinds of separate but interacting knowledge. These would include knowledge of:
(a) story structure
(b) the audience
(c) the author
(d) the cultural context
(e) the rules of the sub-genre

Knowledge of the audience will influence what information should be included in the text and what can be taken as read. Characteristics of the author may lead, for instance, to choices which give a particular perlocutionary force to the text: a moralist might insist on a fictional wrongdoer coming to a sticky end, where a cynic might let him/her go unpunished. The cultural context refers to the socio-historical setting in which the text is produced, which restricts the particular sub-genres available: tales of saints' lives, for instance, all the rage in the Twelfth Century, are now out of vogue and so virtually "untellable". The rules of the sub-genre will place constraints not only on the content of the text, but also on the choice of expressive medium and on stylistic choices within the expressive medium: verse will be fine for a narrative ballad, inappropriate for a detective story. It is knowledge of types (a) and (e) which is encoded in the grammar of the Old French epic described below.

\section{A STORY GRAMMAR FOR THE EPIC}

The study reported in (Pemberton, 1984) is an attempt to identify a general model of story structure, as well as the additional constraints on the form and content of a particular subgenre, the mediaeval French epic. Nine poems composed in mediaeval France, concerning the adventures of a family of French Christian fighters, were analysed and their narrative structure described in terms of a grammar.

It was found necessary to distinguish between various levels of analysis of the text. The textual level is that which the reader experiences directly: in the poems in question, a textual element might be "Guillelmes vit Carlon" (Guillaume saw Charles). The textual level, which is not included in the study, may be thought of as the lexicalised version of the layer of analysis referred to as discourse. This is in tum a modified version of the story line, where the story line is the succession of all the events in a story, and discourse consists of these same events restructured into a form 
suitable for telling. The story line of a detective story, for instance, would recount the crime and its detection in chronological sequence, while the discourse ordering might begin instead with the discovery of the crime. While discourse and story line differ in structure relative to each other, they share the same elements, which consist of propositions formed of events and actors. A typical discourse/storyline element might be "saw(Guillaume, Charles)". The highest level of analysis, the narrative model, is the abstract form of the story line. Units at this level are as free of content as possible, and consist of combinations of functions and roles. The set of functions in the grammar includes elements such as "cause," "succeed" and "attempt", while roles are case-like notions such as "subject," "beneficiary" and "opponent". The narrative model is a construct applicable to many different types of story, whereas units of discourse and storyline will be peculiar to the genre in question.

\section{NARRATIVE}

Information about story structure takes the form of a grammar, whose starting symbol is complex stories. A complex story consists of one or more simple stories. Two stories may be combined using any of four links: these are cause, where the first story causes the second; motive, where a particular action of the first story motivates the second; then, where all the active elements of one story follow all the active elements of the other, and same actor, where there is merely sharing of one or more actors.

The simple story is expanded into an initial situation, an active event, and a final situation. Semantic restrictions on the initial situation state that it must involve two roles, subject and object or object class, in a relation of lack, while the final situation must consist of a negation or restatement of the initial lack. The active event consists of an event in which the subject (or a surrogate) attempts to obtain the object, or a member of the object class. Each element is linked to the next by succession in time, while final situation is linked to the active event in a causal relationship.

The active event is expanded into a five-part structure consisting of motivation, plan, qualification, action, and resolution. The motivation phase consists of the process whereby the subject or surrogate subject acquires the will to bring an end to the initial situation by means of some action. There are two types of motivation: general motivation looks back to the initial situation, while specific motivation looks forward to the action which will end the initial situation. An everyday example will illustrate this distinction: a person who is hungry will have a general motivation to end that state of hunger, but only when confronted with the sight of, say, a loaf of bread, will s/he acquire the specific motivation to end the undesirable state by an action. The stimuli which bring about general and specific motivation are referred to as motivating acts.

The plan phase consists of the subject's acquisition of the knowledge needed to perform the main act: this knowledge is acquired via one or more informing acts. Similarly, qualification is the process of acquiring the power, material or physical, to carry out the main act, via a series of qualifying acts. The action is the subject's attempt to obtain the object, while resolution is the phase in which the success or failure of the action is made clear, determining whether the final situation is to be a negation or a reaffirmation of the initial state. Within this scheme, recursion allows for several attempts to be made on the same goal, or for a new goal to be set.

The terminal elements of this grammar of the narrative model are narrative motifs, which, when instantiated, will make up the chronological flow of the text. It is these motifs which provide the answer to the question "What happened next?" in the story. 
However, few real-life story texts could be described in terms of the bare minimum of propositions generated by the grammar as sketched so far: much more richness of detail is required. The grammar allows for this extra detail by permitting any element of the active event to be supplemented by other narrative motifs, of two types, tied or free. Tied narrative motifs are those which expand elements generated by the narrative model: a qualifying act, for instance, may be expressed in several steps, or several motivating acts may take place. However, even the simplest narrative text contains representations of events which, while not deriving from the narrative model, still describe what happened next (cf. Shen, 1988). These are free narrative motifs, which may illustrate character, exemplify themes, create irony, suggest an historical setting, and so on (cf. Barthes, 1970). Particularly important for the epic is the opposition motif, which serves to hinder, jeopardise or delay some element of the active event.

\section{STORY LINE}

The movement between the elements of the narrative model and those of the story line may be seen essentially as a process of instantiation, detailing which types of actors from the story world of the genre may fill which roles, and which types of events may serve which functions. For reasons of space, the reader is referred to (Pemberton, 1984) for details of the epic story world.

\section{DISCOURSE}

Mapping between story line and discourse involves transformations determining which elements derived from the narrative model may go unexpressed in the text, and which may be presented out of their original order.

Two of the more important deletion transformations involve the non-expression of high level constituents, the plan and qualification phases. These are necessary because few of the stories in the corpus correspond exactly to the the problem solving model of human behaviour reflected in the full form of the grammar. In particular, only two of the simple stories which make up the fifty two stories identified in the texts include an expression of the plan sequence: in both cases this happens when the planned action is a ruse. Thus fifty of the stories are without a planning phase. Moreover, several stories omit both planning and qualification: this happens in stories where an actor who has provided aid to a successful subject is rewarded, or where he is otherwise presented with the object, without having to carry out any directed action to acquire it.

Elsewhere, it is potential redundancy which allows the safe deletion of elements. The initial situation, for instance, is not normally expressed. In cause linked stories, this is presumably because the audience may be assumed to have heard a first story, such as "Charles won Paris from Saladin" and be able to infer from it that Saladin now lacks Paris. In other examples, it is shared cultural assumptions which make it unnecessary to state explicitly that, for instance, where a city is in Saracen hands, a French knight will covet it: to say so would be stating the obvious for the contemporary audience.

Other elements capable of deletion include the resolution and final situation. In all these examples, the principle at work appears to be that of easy inferability: an element of the canonical form may go unexpressed wherever it is almost as easy to retrieve as one expressed in the text (Johnson and Mandler, 1980). The qualifying and action phases are never omitted.

The rules goveming movement of elements will not be discussed in detail here (see Pemberton, 1984). In many stories, the order of elements as they are presented to the reader corresponds exactly to that of the story line, i.e. discourse and story line ordering coincide. Departures from the story line order are associated with the points at which stories are 
interwoven: an example would be when an actor is motivated to pursue one goal while engaged in the pursuit of another. This is a common situation in the texts of the corpus, where a subject often catches sight of his future wife while he is involved in the conquest of a city.

\section{The GESTER Program}

The GESTER program (GEnerating STories from Epic Rules) is a first step towards generating stories from interacting modules of independent knowledge. The program is written in POPLOG Prolog, using Prolog's grammar rule facility. The program has access to information about story structure, in the form of a simplified version of the narrative grammar described above, and to the possible events and actors of the epic sub-genre, in the form of a simplified version of the discourse grammar and a database of objects and attributes in the epic world. In other words, it produces summaries from knowledge sources (a) and (e). No special information about the author and audience is introduced, i.e. the program ignores modules (b) and (c), and I assume that knowledge of the cultural context (d) is adequately represented here by the constraints imposed by the rules of the subgenre (e). Since it would be outside the scope of the project to produce output in the appropriate linguistic form, i.e. Old French verse, the program produces summaries in a makeshift canned English-like representation.

The Story Structure Rules are described at a level of generality which makes it possible for them to be used in the generation of stories of any traditional genre when combined with suitable story world rules and facts. The highest level rules, complex_story, represent the "root" of the parse tree generated by the program to represent the structure of the stories it produces. At the moment, a complex story consists of just two simple stories. The program generates stories by choosing one of the complex_story rules to serve as the root and then proceeding through the tree, making choices as allowed by the constraints imposed by the Story World rules, which in turn refer to the facts stored in the Story World database, until the terminal symbols are reached. Recursion allows for repeated attempts at a goal before final success or failure, and opposition motifs are also generated.

The program uses a variety of features to produce an acceptably coherent story. They may usefully be divided into three groups: firstly, there are story features, which are used to determine a variety of characteristics of the complex story; secondly, role features, which guarantee one kind of coherence by assigning the same roles to the same actors throughout a story; and thirdly, transformation features, which regulate the mapping between story line and discourse levels. There are four story features, Link, Resolution, Mode and Motive. Link is the feature which describes the way in which the two component stories of the complex stories are joined together. Its possible values are cause, motive, then and same_actor, corresponding to the possibilities uncovered in the original study. The resolution feature simply determines whether a story will be successfully resolved, while the mode feature is used to distinguish stories involving friendly interactions from those involving hostility. The motivation feature allows for the specification of particular types of motivating act.

The seven role features specify relations between actors. For instance, there might be a story describing the attack by Guillaume (Subject) on the city of Saragossa (Object, with Object category city), currently ruled by Thibaut (Source), in which he is helped by Charles (Auxiliary), with opposition from Clarion (Opponent), after which Guillaume (Beneficiary) rules the city.

The program also incorporates two transformation features, delete and move, to generate the story summary to the level of discourse. Deletion is managed via the delete 
feature, which may have values delete and no_delete. With delete switched on, some or all of those elements which may legally be deleted will no longer included in the output version. The move feature, with values move and no_move, allows for elements of a second story to be interwoven with those of the first, according to the rules identified in the original study.

The Story Structure Rules use these features to generate complex stories to the level firstly of narrative, then of a simplified form of discourse.

The choice of actors and actions to appear in a story is constrained by the rules contained in the Story World Rules module. These comprise, firstly, "lexicalisation rules" for the non-terminal elements generated by the general grammar, e.g. "try to obtain (a city)" is to be lexicalised as "take by siege" or "take by ruse." Similarly, motivating acts in stories concerning the conquest of a city may be:
(a) hearsay
(b) hearing its defenders are absent
(c) presence of allied prisoners in city
(d) sight of the city
(e) loss of one's own city
(f) a taunt from the city's ruler

When a wife is the object, the subject may be motivated by:
(a) hearsay
(b) a love letter from the woman
(c) nagging by advisors
(d) sight of the woman
(e) hearing a song about the woman

Secondly, Story World Rules detail the restrictions on possible combinations of actors and actions. For instance, while a Christian knight may attempt to marry any Saracen woman, even one who is already married, a Saracen man may not woo a Christian woman, married or not. Other rules specify aspects of loyalty, inheritance, religious belief, marriage and military practice, identified from the analysis of the Old French corpus. Some rules involve several inference steps. For example, to choose an auxiliary, the program first checks to see if any actor is flagged as being particularly friendly towards the subject, as a result of having helped him or having received his help in earlier stories. If not, an auxiliary is sought amongst members of the set of male co-nationals of the appropriate status (e.g. knights rather than commoners or priests), with a family member, father or brother, being preferred to a mere compatriot. However, if the subject has previously displeased the potential auxiliary, perhaps by not rewarding him for services rendered, he will refuse to help. Similarly, if the subject happens to be a rebel, his family and compatriots will all refuse to help him, and he will have to accept an actor of the opposing nation as his ally. None of this reasoning, of course, appears in the story summary itself: it merely serves as its logical underpinning.

The Story World database module contains necessary facts about objects and relations. There are only three types of object represented in the database, people, cities and deities. Facts such as the social status, marital status, family and social relations, nationality and gender are found here, ready to be called on by the Story World Rules. Obviously this is only a very small proportion of the facts which would have to be made available to the rest of the program if it were to produce stories rivalling the richness of incident found in the epic.

The program's output consists of the string of terminal elements making up the content of the story, together with a representation of its parse tree. An example story is set out below. This is a complex story in which the French king, Charles, wins first the city of Narbonne (S1), then a Saracen wife, Blancheflor, whom he sees while invading the city (S2). In order to produce this summary, the program determines possible subject-object combinations for the first story, choosing 
appropriate actors for the supporting roles. At various points in the processing, inferences are made about obligatory and optional events. For instance, Charles, as king, has no need to ask permission of his monarch before setting out to conquer the castle, as a mere knight would. In $\mathrm{S} 1$, the subject and auxiliary are not near the object as the story begins and so must ride to Narbonne, whereas this is unnecessary in S2, since Blancheflor is already in Narbonne. Various events of $\mathbf{S 2}$ are influenced by events of S1: for instance, since Charles forgets to reward his ally, Aymeri, in S1, Aymeri is unwilling to help in the action of S2. In a similar way, while Thibaut opposed Charles in $\mathbf{S 1}$, he cannot do so in $\mathbf{S 2}$, as he is in prison.

The summary was generated with the Link feature instantiated to produce a motive-linked story, and with both deletion and movement options chosen. Elements of the narrative which are deleted because the delete option is chosen have been written in by hand, for the purposes of demonstration.

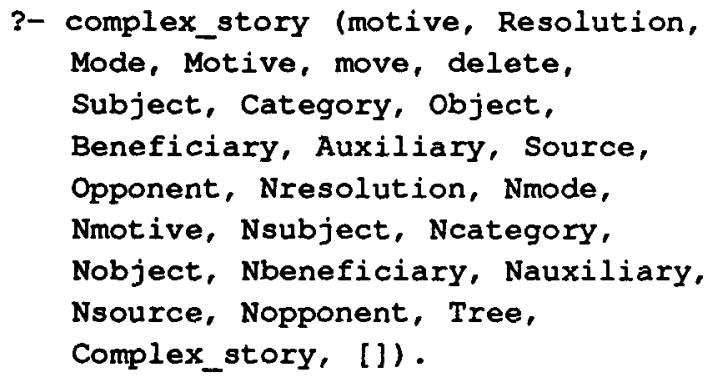

[Then Charles planned to obtain Narbonne for Charles.]

Then Aymeri agreed to help Charles.

Then Charles and Aymeri rode to Narbonne.

[Then Charles was ready to try to obtain Narbonne.]

Then, Charles attacked the walls of Narbonne, currently controlled by Baufumez, helped by Aymeri.
Thibaut and clarion threw burning pitch down on Charles and Aymeri.

Charles and Aymeri retreated.

Then, Charles attacked the walls of Narbonne, currently controlled by Baufumez, helped by Aymeri.

Thibaut and Clarion threw stones down on Charles and Aymeri.

Charles and Aymeri broke into Narbonne.

*As a result of seeing Blancheflor Charles wanted Blancheflor.

Charles succeeded in getting Narbonne. Charles praised God. Charles forgot to reward Aymeri. Charles threw Thibaut into prison.

[Then as a result of getting Narbonne Charles no longer lacked a city.]

Then Charles planned to obtain Blancheflor for Charles.

Then Aymeri refused to help Charles because he was not rewarded.

Then Bertrand agreed to help Charles.

[Charles was now ready to try to obtain Blancheflor.]

Charles abducted Blancheflor, currently controlled by Thibaut helped by Bertrand.

Because Thibaut was in prison he did not oppose Charles and Bertrand.

Clarion opposed Charles and Bertrand in getting Blancheflor.

Charles succeeded in getting

Blancheflor.

Charles praised God.

Charles rewarded Bertrand.

[Then as a result of getting

Blancheflor Charles no longer lacked a wife.]

The following notations are added for clarification:

$$
\begin{aligned}
& * \text { = moved element } \\
& {[]=\text { deleted element }}
\end{aligned}
$$




\section{FUTURE EXTENSIONS}

The work described here represents a first step in implementing a model of story generation. The program now needs to be extended in a number of directions. Firstly, each of the modules must be enriched to enable greater detail, greater variety of incident, and more complexity of structure. The inclusion of a more complex mapping between role and actor, to allow for the distribution among several actors of a single role, will be a first step here. Secondly, the story structure rules will be applied to a representation of a different story world, possibly that of the fairy tale or the romantic short story, in order to test the independence of the modules. A further, more ambitious extension, would be to attempt to incorporate a mapping from the level of discourse, to the textual level: work by Ashby (1976) and Heinemann (1973) indicates such a mapping to be achievable for at least a subset of discourse motifs.

\section{ACKNOWLEDGEMENTS}

My thanks to Aaron Sloman and James Goodlet for comments on an earlier version of this paper.

\section{REFERENCES}

Ashby, Genette. 1976. A Generative Grammar of the Formulaic Language in the Single Combat in the Chanson de Roland. PhD Dissertation: University of Columbia.

Barthes, Roland. 1970. S/Z. Paris: Seuil.

van Dijk, Teun A. 1972. Some Aspects of Text Grammars. The Hague: Mouton.

1980. Macrostructures: An Interdisciplinary Study of Global Structures in Discourse, Interpretation and Cognition. New York: Lawrence Erlbaum.

Garnham, Alan. 1983. What's wrong with story grammars. Cognition, 15: 145-54.

Heinemann, Edward A. 1973. Composition stylisee et technique litteraire dans la
Chanson de Roland. Romania, 94: 1-27.

Johnson, Nancy S. and Jean M. Mandler. 1980. A Tale of Two Structures: underlying and surface forms in stories. Poetics, 9: 51-86.

Mandler, Jean M and Nancy S. Johnson. 1980. On Throwing Out the Baby with the Bathwater: A Reply to Black and Wilensky's Evaluation of Story Grammars. Cognitive Science, 4: 305312.

Mann, William. 1984. Discourse Structures for Text Generation. COLING-84: 367-375.

Pemberton, Lyn. 1984. Story Structure: A Narrative Grammar of Nine Chansons de Geste of the Guillaume d'Orange Cycle. PhD dissertation: University of Toronto.

1987. A Story Grammar for the Old French Epic. MSc dissertation: University of Sussex.

Propp, Vladimir. 1928, 1968. Morphology of the Folktale. Austin, Texas: University of Texas Press.

Rumelhart, David E. 1975. Notes on a schema for stories. In D.G. Bobrow and A.M. Collins, eds, Representation and Understanding. New York: Academic Press.

Ryan, Marie-Laure. 1981. On the Why, What and How of Generic Taxonomy. Poetics, 10: 109-126.

Shen, Yeshayahu. 1988. Schema Theory and the Processing of Narrative Texts: the $\mathrm{X}$-bar Story Grammar and the notion of Discourse Topic. Journal of Pragmatics, 12: 639-676.

Wilensky, Robert. 1983. Story grammars versus story points. Behavioral and Brain Sciences, 6: 579-623.

Yazdani, Masoud. 1982. How to write a story. ECAI-82: 259-60. 